\title{
Crescimento, eficiência fotossintética e eficiência do uso da água em quatro espécies de leguminosas arbóreas tropicais
}

\author{
Javier Alberto Pinzón-Torres ${ }^{1}$ e Marlene Aparecida Schiavinato ${ }^{1,2}$
}

Recebido: 17.04.2008; aceito: 21.08.2008

ABSTRACT - (Growth, photosynthetic and water use efficiency in four tropical tree legume species of). The series of studies covering variables of growth and photosynthetic parameters helps to clarify the relationship between individuals of a plant community. The objective of this study was to evaluate the growth, photosynthetic and water use efficiency in four legume trees in a period of three months under conditions of a greenhouse. It was observed that Myroxylon peruiferum showed higher values for the rate of relative growth, the ratio of leaf area and the mass leaf and greater leaf specific area, compared with the other three species. But, this species did not presente the highest photosynthetic rate, as consequence of increased investment in leaf area. All species, except Lonchocarpus muehlbergianus showed the same efficiency in the use of water. Although M. peruiferum does not fix atmospheric nitrogen through symbiosis with rhizobia, it seems to be an efficient species to use the nitrogen available in the soil for the production of biomass.

Key words: Centrolobium tomentosum, Inga vera, Lonchocarpus muehlbergianus, Myroxylon peruiferum

RESUMO - (Crescimento, eficiência fotossintética e eficiência do uso da água em quatro espécies de leguminosas arbóreas tropicais). O conjunto de estudos que abrangem variáveis de crescimento e parâmetros fotossintéticos ajuda a esclarecer as relações entre os indivíduos de uma comunidade vegetal. O objetivo deste trabalho foi avaliar o crescimento, a eficiência fotossintética e a eficiência no uso da água em quatro leguminosas arbóreas, durante três meses, sob condições de casa de vegetação. Foi observado que Myroxylon peruiferum apresentou os maiores valores quanto à taxa de crescimento relativo, razão de área foliar e de massa foliar e maior área foliar específica, quando comparada com as outras três espécies. Porém, não apresentou a maior taxa fotossintética, como consequência do maior investimento em área foliar. Todas as espécies, exceto Lonchocarpus muehlbergianus apresentaram a mesma eficiência do uso da água. Embora M. peruiferum não fixe nitrogênio atmosférico por meio de simbiose com rizóbios, se observou que é uma espécie eficiente para aproveitar o nitrogênio disponível no solo para a produção de biomassa.

Palavras-chave: Centrolobium tomentosum, Inga vera, Lonchocarpus muehlbergianus, Myroxylon peruiferum

\section{Introdução}

Na composição e estrutura das florestas tropicais, as leguminosas apresentam uma alta diversidade de gêneros e espécies (Moreira et al. 1992). Não obstante, estas espécies devem se adaptar morfológica e fisiologicamente às diversas condições ambientais para determinar a sua competitividade dentro da comunidade vegetal.

As adaptações morfológicas variam segundo a alocação da biomassa, que pode se manifestar pelo incremento em massa, volume, comprimento ou área das diferentes estruturas da planta, o que é avaliado como crescimento (Gloser \& Gloser 1996).

Quanto às adaptações fisiológicas, Hall et al. (1993) mencionam que existem quatro fatores relacionados à fotossíntese que influenciam no incremento da biomassa: (i) a quantidade de luz incidente, que é determinada pelo clima; (ii) a proporção da luz interceptada pelas organelas, que depende da estrutura e da cor das partes que formam a planta; (iii) a eficiência da conversão da luz interceptada pelas organelas, que é determinada diretamente pelos processos fotossintéticos, expressando a relação direta entre a produtividade e a fotossíntese e; (iv) a perda de biomassa pela respiração, com finalidade de manter e formar constantemente novos tecidos.

Qualquer que seja o tipo de adaptação, morfológico ou fisiológico, irá influenciar a planta como um todo. Deste modo, o incremento da biomassa vegetal, avaliado como taxa de crescimento relativo (TCR), ocorre em função de um componente morfológico,

1. Universidade Estadual de Campinas, Instituto de Biologia, Departamento de Fisiologia Vegetal, Caixa Postal 6109, 13083-970 Campinas, SP, Brasil

2. Autor para correspondência: mschiavi@unicamp.br 
a Razão de Área Foliar (RAF), que é área foliar por unidade de massa seca total, e de um componente fisiológico, a Taxa de Assimilação Líquida (TAL), que é o incremento de massa seca total em função da área foliar (Wright \& Westoby 2000).

ARAF varia em função da Área Foliar Específica (AFE), que é a área foliar por unidade de massa seca foliar, e da Razão de Massa Foliar (RMF), que é a proporção de massa seca total alocada às folhas. Estes parâmetros são relativamente sensíveis às mudanças ambientais, embora os incrementos na RAF sejam mais conseqüências do incremento da AFE do que da RMF, já que os valores da AFE são mais sensíveis às alterações da irradiância (Lambers \& Poorter 1992). Segundo Meziane \& Shipley (1999), o grau das diferenças na taxa de crescimento relativo (TCR) dentre as espécies, mesmo cultivadas em idênticas condições ambientais, é o que influencia a dinâmica e a estrutura de uma comunidade vegetal.

A AFE é uma medida estratégica da alocação da biomassa que reflete a área disponível para a captura de luz por unidade de fotoassimilados investidos nas folhas (Marañon \& Grubb 1993). Para alguns autores, a AFE seria o parâmetro que melhor explicaria as diferenças na TCR, entre espécies vegetais.

A TCR pode estar associada com a taxa de assimilação líquida (TAL), no sentido de relacionar a taxa fotossintética à quantidade de área foliar disponível para a interceptação de luz e à alocação da biomassa foliar (Lambers et al. 1998), o que significa, o ganho de carbono através da fotossíntese e a perda de carbono através da respiração (James \& Rebecca 2007). Alguns autores consideram que há pouca ou nenhuma relação entre TCR e TAL e sugerem que as variações da TCR ocorram principalmente pelas diferenças na AFE (Lambers \& Poorter 1992).

As variações na TCR também são influenciadas pela disponibilidade de nitrogênio $(\mathrm{N})$, pois em condições adequadas de nutrição as plantas apresentam altos valores de TCR (Meziane \& Shipley 1999, Wright \& Westoby 2000). Deste modo, as espécies com altas TCR apresentam altas concentrações de N orgânico por unidade de massa seca, o que sugere uma alta eficiência no uso de $\mathrm{N}$ no processo de fixação de carbono (Poorter et al. 1990).

Wright \& Westoby (2000) mencionam que a TCR também poderia variar em função da produção do $\mathrm{N}$ foliar, portanto, do incremento de massa por unidade de $\mathrm{N}$ foliar. Estes autores enfatizaram que, assim como a TAL, a produtividade e a concentração do $\mathrm{N}$ foliar indicam atividade foliar, e por este motivo estão correlacionadas com as taxas de fotossíntese e de respiração, com a partição do $\mathrm{N}$ foliar para o metabolismo, para a formação de componentes estruturais e com a alocação de biomassa.

Por outro lado, a taxa fotossintética refletida indiretamente na TAL é o resultado da interação das variações dos fatores ambientais (luz, $\mathrm{CO}_{2}$, temperatura, disponibilidade de água e nutrientes, idade das folhas, etc.), com as diferenças específicas de cada espécie, segundo seu genótipo (Lambers et al. 1998). Neste sentido, vários estudos envolvendo trocas gasosas têm demonstrado as diferenças nas taxas fotossintéticas das diferentes espécies sob as mesmas condições naturais (Ishida et al. 1999).

A razão entre a taxa fotossintética e a taxa de transpiração resulta na eficiência do uso da água (EUA), que é mais um parâmetro fisiológico que expressa quantitativamente o comportamento momentâneo das trocas gasosas na folha e que varia entre e dentro das espécies vegetais (Larcher 2000).

O conjunto de estudos que abrangem variáveis de crescimento e parâmetros fotossintéticos ajuda a esclarecer as relações entre os indivíduos de uma comunidade vegetal e são úteis para projetos de conservação, proteção e recuperação de áreas. Por tal motivo, o presente trabalho teve como objetivo avaliar o crescimento, a eficiência fotossintética e a eficiência do uso da água de quatro espécies de leguminosas arbóreas tropicais, sob condições de casa de vegetação e associar estes parâmetros com o estádio de sucessão destas espécies.

\section{Material e métodos}

Material vegetal e condições de cultivo - Foram cultivadas plantas de Centrolobium tomentosum Mart. ex Benth., Inga vera Willd., Lonchocarpus muehlbergianus Hassl. e Myroxylon peruiferum L.f., a partir de sementes coletadas na Reserva Municipal de Santa Genebra, que corresponde a um fragmento da Mata Atlântica, localizada no Município de Campinas,

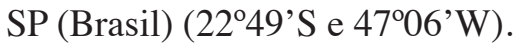

Estas espécies foram mantidas em vasos de $5 \mathrm{~L}$, contendo solo proveniente da mesma Reserva, sob condições de luz e temperatura da casa de vegetação do Instituto de Biologia da Universidade Estadual de Campinas. Todas as espécies, exceto $M$. peruiferum que não forma nódulos radiculares, foram previamente inoculadas com isolados de rizóbios nativos provenientes do solo da Reserva de Santa Genebra. Todas as plantas foram irrigadas com solução 
nutritiva de Hoagland \& Arnon (1950), duas vezes por semana, usando $1 / 4$ da força iônica de nitrato $\left(\mathrm{NO}_{3}{ }^{-}=\right.$ $3,75 \mathrm{mM}$ ) como fonte de nitrogênio.

Variáveis de crescimento - Aos sete e dez meses de idade, 15 plantas de cada uma das espécies foram selecionadas, coletadas e separadas em lâminas foliares, caule + pecíolos e raízes. Após determinação da área foliar (AF), com um integrador de área (LiCor, modelo Li-300), todo o material foi colocado para secagem em estufa (Fanem) a $60{ }^{\circ} \mathrm{C}$ com ventilação forçada por $48 \mathrm{~h}$, até obtenção de massa de matéria seca constante (MS) em balança analítica (Marte precisão de três casas decimais).

Os dados de MS e AF obtidos foram utilizados para obtenção dos parâmetros de crescimento, segundo Hunt (1982): no intervalo de três meses, entre sete a dez meses de idade, se calculou a taxa de crescimento relativo $\left(\mathrm{TCR}=\left(\ln \mathrm{MS}_{2}-\ln \mathrm{MS}_{1}\right)\right.$ $\left.\left(t_{2}-t_{1}\right)^{-1}\right)$ em mg g ${ }^{-1}$ dia $^{-1}$ e a taxa de assimilação líquida $\left(\mathrm{TAL}=\left(\left(\mathrm{MS}_{2}-\mathrm{MS}_{1}\right)\left(\ln \mathrm{AF}_{2}-\ln \mathrm{AF}_{1}\right)\left(\mathrm{t}_{2}-\right.\right.\right.$ $\left.\left.\mathrm{t}_{1}\right)^{-1}\left(\mathrm{AF}_{2}-\mathrm{AF}_{1}\right)^{-1}\right)$ em $\mathrm{mg} \mathrm{cm} \mathrm{cia}^{-1}$. Para três e dez meses de idade, respectivamente, calculou-se a razão de área foliar $\left(\mathrm{RAF}=\mathrm{AF} \mathrm{MS} \mathrm{total}{ }^{-1}\right) \mathrm{em} \mathrm{cm}^{2} \mathrm{~g}^{-1}$, área foliar específica $\left(\mathrm{AFE}=\mathrm{AF}\right.$ MS de folhas $\left.{ }^{-1}\right) \mathrm{em} \mathrm{cm}^{2}$ $\mathrm{g}^{-1}$, razão de massa foliar $(\mathrm{RMF}=\mathrm{MS}$ de folhas MS total $^{-1}$ ) e razão raíz/parte aérea $=$ MS de raiz MS de parte aérea ${ }^{-1}$. Onde: $\mathrm{MS}=$ massa seca, $\mathrm{MS}_{(1)}=$ massa seca total no tempo $1(\mathrm{~g}, \mathrm{mg}), \mathrm{MS}_{(2)}=$ massa seca total no tempo $2(\mathrm{~g}, \mathrm{mg}), \mathrm{AF}_{(1)}$ = área foliar total no tempo $1\left(\mathrm{~cm}^{2}\right), \mathrm{AF}_{(2)}=$ área foliar total no tempo 2 $\left(\mathrm{cm}^{2}\right), \mathrm{t}_{1}=$ coleta no tempo $1($ dias $), \mathrm{t}_{2}=$ coleta no tempo 2 (dias).

Determinação do nitrogênio total - Amostras de folhas, caules e raízes das plantas com sete e dez meses de idade foram secas em estufa a $60{ }^{\circ} \mathrm{C}$ e posteriormente moídas em moinho de bola para determinação de nitrogênio total (Nt). Utilizou-se a técnica semimicro Kjeldahl, seguida por destilação a vapor e titulação para quantificação de amônio. Com base na MStotal e no $\mathrm{Nt}$ foi calculada a razão de eficiência do nitrogênio (REN), para expressar a quantidade de MS produzida por unidade de Nt (Poorter et al. 1990): REN = (MS total $\times 1000)(\mathrm{Nt})^{-1} \mathrm{em} \mathrm{g} \mathrm{mg}^{-1}$.

Eficiência fotossintética - A taxa fotossintética $(A)$ foi registrada, em $\mu \mathrm{mol} \mathrm{CO}_{2} \mathrm{~m}^{-2} \mathrm{~s}^{-1}$, como taxa líquida de trocas de $\mathrm{CO}_{2}$ por unidade de área foliar utilizando um Sistema de Fotossíntese Portátil - IRGA (Infra-Red Gas Analyser) (LCA-4 ADC, Analytical Development Company Limited).
As medições foram realizadas no período de maio a junho de 2001, de 9:00 a 14:00 h, utilizando cinco plantas por espécie. Esse intervalo de tempo foi utilizado visto que, segundo Hall et al. (1993), as curvas fotossintéticas de várias espécies apresentam a máxima $A$ no período da manhã e atingem o estado de saturação após este período. As medidas foram realizadas na última folha completamente desenvolvida, registrando três medições para cada folha a intervalos de 10 a 15 s. Simultaneamente obteve-se a radiação fotossintéticamente ativa $(P A R)$ em $\mu \mathrm{mol}$ fótons $\mathrm{m}^{-2} \mathrm{~s}^{-1}$ e a taxa de transpiração $(E)$ em mmol $\mathrm{m}^{-2} \mathrm{~s}^{-1}$.

Para avaliar quantitativamente a $A$ e a $E$ no período de tempo estipulado, as curvas de tendência foram submetidas à regressão polinomial de $2^{\mathrm{a}}$ ordem e calculadas as integrais definidas pelas correspondentes equações (Flemming \& Gonçalves 1992). Para cada caso foi calculada a área delimitada pela curva $y=f(x)$ (onde $f=P A R, A$ ou $E$ e $x=$ tempo). Foram tomados como intervalos de integração $a$ (1) como limite inferior e $b(5)$ como limite superior, que correspondem às faixas dos 5 registros realizados de 9:00 a 14:00 h. Com a magnitude resultante do cálculo da integração, se compararam as quatro espécies em função do tempo.

A razão $A / E$, utilizada por Larcher (2000) para calcular a eficiência do uso da água (EUA), foi aplicada para as espécies em estudo, porém utilizando os valores integrados de $\int A$ e $\int E$, respectivamente: $\mathrm{EUA}=\int \mathrm{A} \int \mathrm{E}^{-1} \mathrm{em} \mathrm{mmol} \mathrm{CO} \mathrm{mol} \mathrm{H}_{2} \mathrm{O}^{-1}$.

Extração de clorofilas totais - Após o registro das variáveis fotossintéticas, discos foliares (das mesmas folhas) foram utilizados para extração de pigmentos fotossintéticos utilizando-se dimetil-sulfóxido (DMSO), segundo Hiscox \& Israelstam (1978). As leituras da densidade óptica foram determinadas em espectrofotômetro (Pharmacia Biotech. Ultrospec 1000) a 663 e $645 \mathrm{~nm}$. Discos foliares com as mesmas áreas foram submetidos à secagem, por $48 \mathrm{~h}$ em estufa a $80{ }^{\circ} \mathrm{C}$, para obtenção da MS. Os cálculos foram expressos em mg $\mathrm{g} \mathrm{MS}^{-1}$ foliar, de acordo com as equações de Arnon (1949) e Lichtenthaler (1987): Clor. total $=\left(\left(20,2 \mathrm{xA}_{645}+8,02 \mathrm{xA}_{663}\right) \cdot \mathrm{V}\right)(1000 \mathrm{MS})^{-1}$ em mg gMS ${ }^{-1}$. Onde: $\mathrm{A}_{645}=$ absorbância a $645 \mathrm{~nm}$, $\mathrm{A}_{663}=$ absorbância a $663 \mathrm{~nm}, \mathrm{~V}=$ volume da amostra $(\mathrm{mL}), \mathrm{MS}=$ massa seca da $\operatorname{amostra}(\mathrm{g})$.

Análise estatística - Os dados foram analisados utilizando uma análise de variância simples, seguida do cálculo da diferença mínima significativa (DMS) pelo teste de Tukey a $5 \%$ de probabilidade. Foi 
utilizado o programa estatístico BioEstat 3.0 (Ayres et al. 2003).

\section{Resultados e Discussão}

Durante o período de cultivo, a temperatura da casa de vegetação variou entre $15,2{ }^{\circ} \mathrm{C}$ (mínima) e $37,2{ }^{\circ} \mathrm{C}$ (máxima). A temperatura média registrada diariamente às 9:00 $\mathrm{h}$ foi de $28,6{ }^{\circ} \mathrm{C} \pm 2,4$. Nestas condições, observou-se que no intervalo de três meses de crescimento a maior TCR foi a de $M$. peruiferum (figura 1A). Igualmente, esta espécie apresentou incrementos na RAF (figura 1B) e na AFE (figura 1C) aos 7 e 10 meses de idade e a maior RMF em ambas as idades (figura 1D), quando comparada com as outras espécies.

A maior TCR em M.peruiferum indica incremento de MS e de AF. Porém, alguns autores mencionam que a RMF não é um parâmetro suficiente para explicar uma alta TCR, porque não é a proporção da biomassa investida nas folhas que determina a TCR, mas sim a quantidade de AF determinada pela unidade de MS total (RAF) ou pela unidade de MS foliar (AFE) (James \& Rebecca 2007).

Tem-se observado que em várias espécies herbáceas e lenhosas, as baixas densidades do tecido foliar e, por conseguinte, a alta AFE, geralmente acompanha alta TCR (Reich et al. 1992). Desta maneira, existe correlação entre crescimento e taxa de expansão da AF e as espécies com alta AFE e alta TCR, aparentemente produzem folhas com baixo investimento em biomassa. Portanto, segundo Antúnez et al. (2001), as diferenças na AFE entre espécies se associam ao espessamento foliar determinado pelo genótipo.

Wang et al. (1994) indicam que as alterações nos parâmetros relacionados à morfologia foliar, como RAF, RMF e AFE, observados com o aumento da idade das plantas, revelam a capacidade de adaptação das folhas às condições variáveis da radiação ao longo do crescimento e desenvolvimento da planta. Por tal motivo, o incremento da AF é uma medida estratégica para maximizar a superfície da folha, refletindo a área disponível para a absorção de luz por unidade de fotoassimilado investido (Marañon \& Grubb 1993). Dessa forma, e concordando com Antúnez et al. (2001), a maior TCR de M. peruiferum, pode ser explicada pelo incremento da AF, evidenciado pelas variações na RAF (figura 1B) e na AFE (figura 1C) entre 7 e 10 meses de idade, já que esta espécie não apresentou incremento na RMF nesse mesmo período, embora a RMF tenha sido alta nas duas idades (figura 1D). Segundo Grotkopp \& Rejmánek (2007), uma alta AFE permite maior eficiência na captura da energia solar e é a partir da fotossíntese, em condições nutricionais ótimas, que a capacidade competitiva se evidencia.

A RAF, a AFE e a RMF de $C$.tomentosum e de $I$. vera diminuíram de 7 para 10 meses de idade (figura 1B, C, D), significando perda de biomassa foliar. Esta perda aconteceu devido à época relativamente seca e fria em que foram realizadas as medições (maio, junho), pois se tratam de espécies decíduas, cuja característica principal é perder as folhas nesta época do ano (Lorenzi 1992). Lambers et al. (1998) comentam que quando a AFE diminui, também diminui a quantidade de AF disponível para a interceptação da luz e, por conseguinte, diminuem os ganhos líquidos de carbono, refletindo-se na TCR.

A espécie L.muehlbergianus apresentou a menor TCR (figura 1A) porque se trata de uma espécie climáxica, cuja principal característica é o crescimento lento. Além disso, esta espécie investiu menos biomassa na parte aérea e mais biomassa na parte radicular (figura 1F), corroborando as observações realizadas por Cancian \& Cordeiro (1998) que mencionam que L. muehlbergianus é uma espécie que se caracteriza por apresentar maior acúmulo de MS nas raízes que na parte aérea. Reich et al. (1992) comentam que a razão da massa radicular poderia ser mais um parâmetro de alocação de biomassa relacionada à TCR, mas no presente estudo este parâmetro não foi determinante para que L.muehlbergianus apresentasse a maior TCR, quando comparada com M. peruiferum.

A TCR pode estar associada com a TAL, no sentido de refletir a taxa fotossintética na quantidade de AF disponível para a interceptação de luz e na alocação da biomassa foliar (Lambers et al. 1998), mas alguns autores (Lambers \& Poorter 1992, Wright \& Westoby 2000), comentam que há pouca ou nenhuma relação entre TCR e TAL e que é de se esperar que as variações da TCR ocorram principalmente em função das diferenças na AFE. Esta apreciação pode ser corroborada observando-se os valores da TAL das espécies estudadas, onde $M$. peruiferum e I. vera foram estatisticamente iguais e maiores do que a TAL de C. tomentosum e L. muehlbergianus (figura 1E). No entanto, M. peruiferum apresentou maior TCR do que I. vera.

As variações na TCR também ocorrem pela disponibilidade de nutrientes, pois em condições adequadas de nutrição, as plantas apresentam altos 

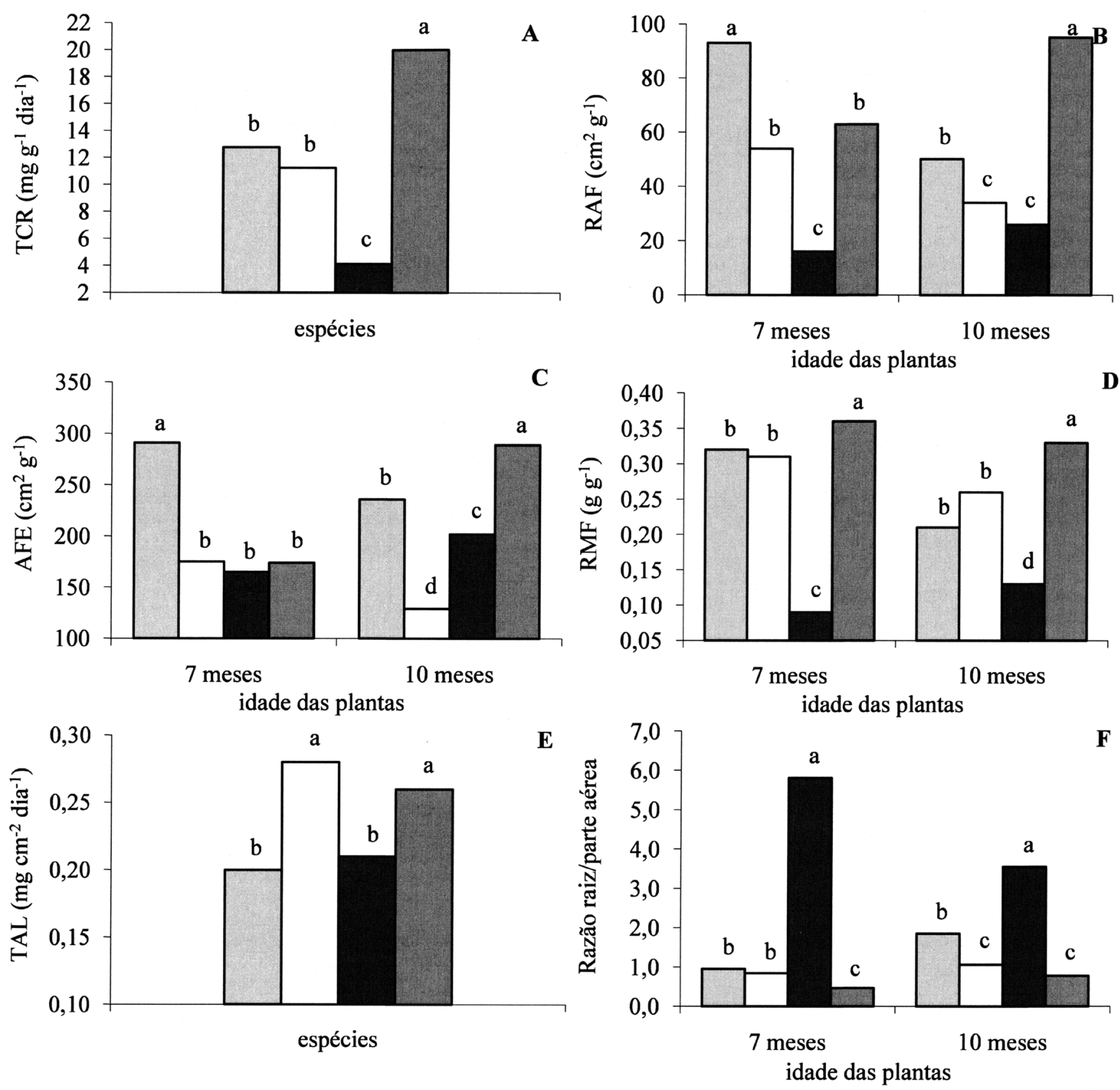

Figura 1. Variáveis de crescimento. Taxa de crescimento relativo (TCR) (A), razão de área foliar (RAF) (B), área foliar específica (AFE) (C), razão de massa foliar (RMF) (D), taxa de assimilação líquida (TAL) (E), razão raíz/parte aérea (F) das espécies Centrolobium tomen-

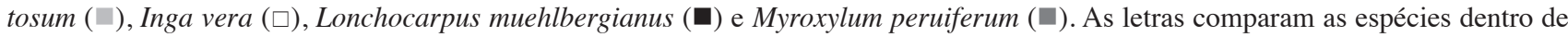
cada idade ou crescimento em um intervalo de 3 meses (TCR e TAL), pelo teste de Tukey ao nível de 5\% probabilidade ( $\mathrm{n}=4$ espécies $\times 15$ repetições). 
valores de TCR (Lambers \& Poorter 1992). As espécies com altas TCR apresentam altas concentrações de Nt por unidade de MS total alocando mais $\mathrm{N}$ para as folhas e indicando melhor uso deste $\mathrm{N}$ na fixação de carbono (Poorter et al. 1990).

A maior razão final/inicial de Nt entre 7 e 10 meses de idade foi apresentada por M. peruiferum e, por conseguinte, maior REN com valores que diferem significativamente quando comparados com os das outras espécies (figura 2A, B). Poorter et al. (1990) comentam que quanto maior o quociente de eficiência, maior é a utilização do $\mathrm{N}$ na produção de MS e Wright $\&$ Westoby (2000) comentam que o incremento de MS se deve ao incremento de $\mathrm{N}$ foliar e à concentração de $\mathrm{N}$ por unidade de AF, porém, existe variabilidade nas leguminosas quanto à assimilação de $\mathrm{N}$ absorvido e assimilado, pois este $\mathrm{N}$ pode ser rapidamente translocado para estruturas novas, favorecendo em distintos graus o desenvolvimento vegetativo (Brown \& Walsh 1994).

Espécies com altas TCR e AFE são caracterizadas por apresentarem altas concentrações de $\mathrm{N}$ acumulado. Desta maneira, quando uma espécie de rápido crescimento acumula mais $\mathrm{N}$ por unidade de MS, aloca mais biomassa para as folhas, usando mais eficientemente este $\mathrm{N}$ na fotossíntese (Poorter et al. 1990,Lambers \& Poorter 1992). Embora M.peruiferum não fixe $\mathrm{N}$ atmosférico por meio de nódulos, se observou que é uma espécie muito eficiente em utilizar o N disponível para a produção de biomassa, refletindose na sua maior REN (figura 2B).

Alguns autores encontram correlação positiva entre REN e AFE, porque as variações interespecíficas na AFE podem determinar as diferenças no conteúdo
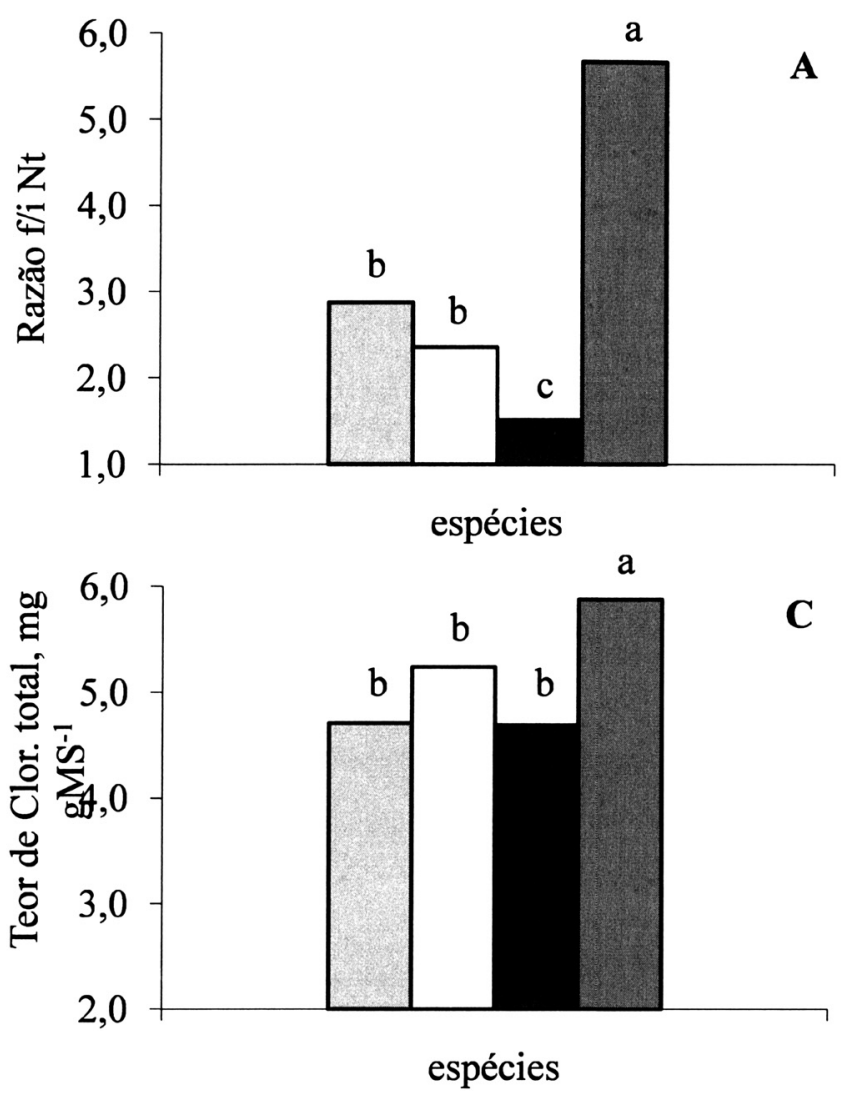
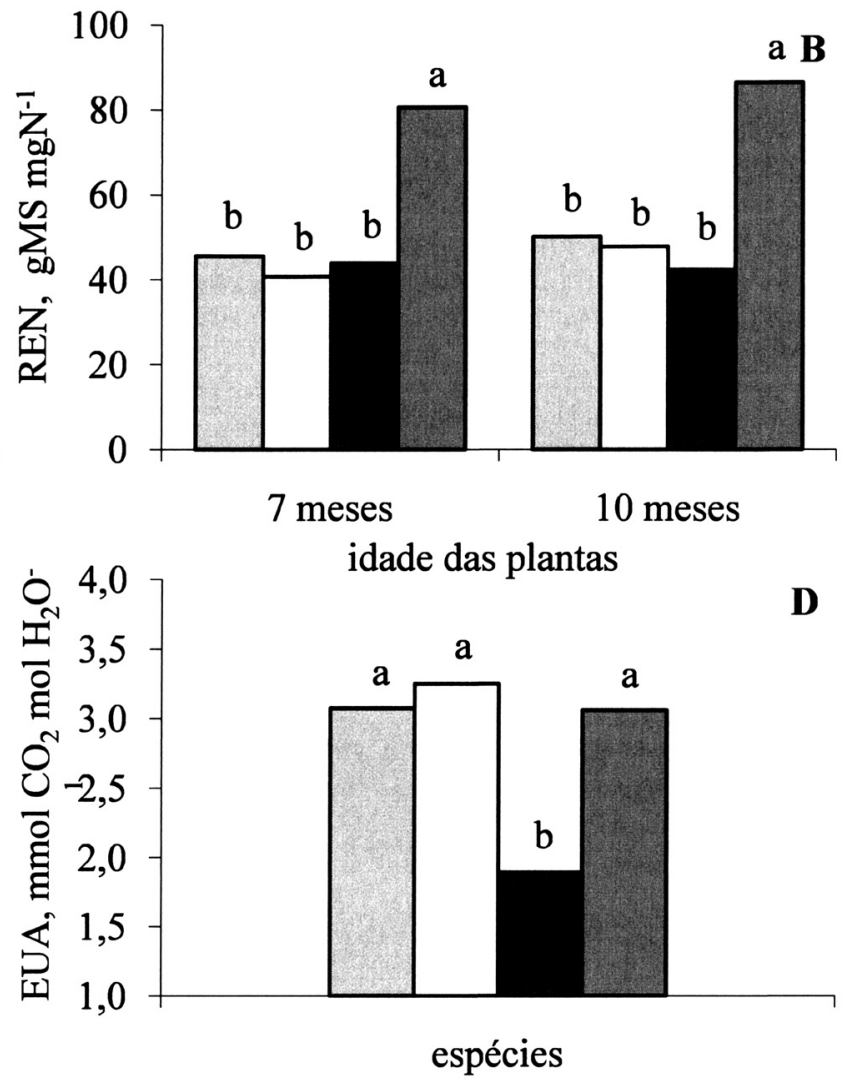

Figura 2. Aproveitamento do nitrogênio. Razão final/inicial do nitrogênio total (A), razão da eficiência do nitrogênio (REN) aos 7 e 10 meses de idade (B). Teor de clorofilas totais por unidades de massa seca foliar aos 10 meses de idade (C). Eficiência no Uso da Água (EUA) (D) das espécies Centrolobium tomentosum ( $\square$ ), Inga vera ( $\square$ ), Lonchocarpus muehlbergianus ( $\square$ ) e Myroxylum peruiferum ( $\square$ ). As letras comparam as espécies dentro de cada idade ou crescimento em um intervalo de 3 meses (Rf/i Nt), pelo teste de Tukey ao nível de $5 \%$ probabilidade ( $\mathrm{n}=4$ espécies $\mathrm{x} 15$ repetições). 
de $\mathrm{N}$ orgânico expressado por unidade de AF (Schieving \& Poorter 1999). Segundo Garnier et al. (1995), a eficiência pela qual uma espécie utiliza $\mathrm{N}$ para crescer, ou seja, uma correlação positiva entre REN e AFE, se dá pelos seguintes aspectos: diferenças entre as espécies na absorção da luz nas folhas, variações na proporção de compostos de $\mathrm{N}$ orgânico alocado pela fotossíntese, funções não fotossintéticas, diferenças nos tempos de assimilação de $\mathrm{CO}_{2}$ por unidade de $\mathrm{N}$ fotossintético, diferenças na quantidade de fotorespiração e pelas diferenças no conteúdo de $\mathrm{N}$ por unidade de AF. Espécies com altas REN sugerem alto investimento do seu $\mathrm{N}$ para a maquinária fotossintética e isto significa maior conteúdo de $\mathrm{N}$ tilacoidal, maior taxa de transporte de elétrons por unidade de clorofila e maior quantidade de Rubisco (Wright \& Westoby 2000). Neste sentido, $M$. peruiferum que também apresentou maior teor de clorofilas totais, corrobora a correlação positiva entre produtividade de $\mathrm{Nt}$ e investimento deste $\mathrm{N} \mathrm{em}$ pigmentos fotossintéticos (figura 2C).

Por todos os resultados obtidos na avaliação do crescimento, e com $\int P A R$ igual para todas as espécies (figura 3A), era de se esperar que $M$. peruiferum tivesse a maior taxa fotossintética $(A)$, já que o maior investimento de AF por unidade de biomassa providenciaria um maior retorno no investimento de carbono, no entanto, isso não foi observado quando foi integrada a equação polinomial de $2^{\mathrm{a}}$ ordem da curva de tendência da $A$ (figura $3 \mathrm{~B}$ ). A integração destes polinômios em função do tempo se constituiu numa ferramenta matemática propicia para analisar e comparar o grau de aproveitamento da $P A R$ e da $A$ das espécies em estudo.

Zelitch (1982) comenta que a produtividade vegetal depende do uso dos fotoassimilados, que podem ser utilizados para aumentar a capacidade fotossintética por meio do incremento da $\mathrm{AF}$ e da produção de clorofila ou ser armazenados como biomassa. Em $M$. peruiferum, parece que o crescimento foi refletido no incremento da $\mathrm{AF}$ ( $\mathrm{RAFe} \mathrm{AFE}$ ) e produção de clorofila, embora a $\int A$ não tenha sido a maior em relação às outras espécies. Não obstante, observações realizadas com outras espécies indicam que não há correlação entre $A$ e $\mathrm{AF}$, devido à plasticidade fenotípica da $A$ por unidade de $\mathrm{AF}$, mas que pode existir correlação entre $A$ e MS foliar, devido à relação causa-efeito quanto ao no ganho diário de biomassa (Poorter et al. 1990, Kitajima 1994). No entanto, a maior RMF para M. peruiferum aos 7 e 10 meses de idade também não foi refletida em maior $\int A$.
Os maiores valores de $\int E$ também foram observados para I. vera (figura 3B), porém, todas as espécies, exceto L. muehlbergianus, apresentaram as mesmas EUA (figura 2D). Schulze \& Hall (1982) comentam que a vantagem ecológica que as plantas obtêm ao variar a $A$ é manter uma relação linear com a EUA, otimizando assim as trocas gasosas.

As variáveis de crescimento podem caracterizar os estádios de sucessão das espécies. As espécies $M$. peruiferum e L. muehlbergianus estão catalogadas como climáxicas, enquanto que $C$. tomentosum e $I$. vera estão catalogadas como pioneiras (Lorenzi 1992). Uma espécie climáxica ou de sucessão tardia é aquela que consegue se instalar na substituição seqüencial da sucessão no interior da floresta, apresentando crescimento lento e geralmente tolerando a sombra. Entre as características ecológicas de uma espécie pioneira ou de sucessão inicial estão: apresentar maior altura, maior crescimento em extensão do caule, maior produção de folhas e maior TCR que as espécies climáxicas (Ramakrishnan et al.1982). Estas espécies colonizam primeiramente os ambientes perturbados porque se desenvolve bem a pleno sol.

No presente estudo, em condições de casa de vegetação, M. peruiferum apresentou as características próprias de uma espécie pioneira. Porém, Souza \& Válio (2001) comentam que $M$. peruiferum destacou-se pelos baixos potenciais de aumento nas variáveis de MS, AF, TCR e TAL, tanto em condições sombreadas como não sombreadas e os mesmo autores caracterizaram esta espécie como climáxica.

Segundo Antúnez et al. (2001), as espécies arbóreas decíduas apresentam maiores TCR, AFE e $A$ que as espécies sempre-verdes. Estes mesmos autores observaram que a maior TCR em espécies decíduas pode estar determinada pela maior RAF, que por sua vez é estimada pela maior AFE, e que outras características, como RMF e TAL avaliam em menor escala a variação da TCR. Deste modo, a maior TCR das espécies decíduas é considerada como uma determinante na distribuição em hábitats, favorecendo a capacidade competitiva. Não obstante, estes autores enfatizam que não se pode generalizar afirmando que as espécies decíduas sempre crescerão mais rapidamente que as espécies sempre-verdes. Isso poderia explicar o fato de $M$. peruiferum, mesmo sendo climáxica, ter apresentado características de pioneira.

Por outro lado, espécies intolerantes à sombra parecem apresentar maiores RAF, AFE, RMF e TAL, que espécies tolerantes (Kitajima 1994). Deste modo, 


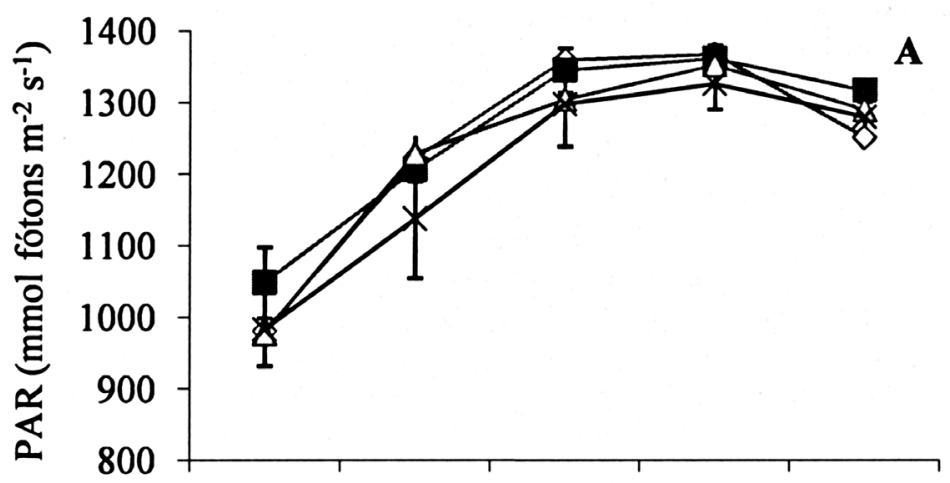

C. tomentosum: $\mathrm{y}=-60,316 \mathrm{x} 2+430,86 \mathrm{x}+606,67$;

$\mathrm{R} 2=0,9994 ; \int \Omega=18374,2\left(\mathrm{~mol}_{\text {fótons }} \mathrm{m}^{-2}\right)$ a

I. vera: $\mathrm{y}=-37,557 \mathrm{x}^{2}+294,74 \mathrm{x}+784,44$;

$R^{2}=0,9917 ; \int \Omega=18440,2\left(\right.$ mol fótons. $\left.m^{-2}\right) a$

L. muehlbergianus: $\mathrm{y}=-37,557 \mathrm{x} 2+294,74 \mathrm{x}+784,44 ; \mathrm{R} 2$

$=0,9917 ; \int \Omega=18440,2\left(\mathrm{~mol}_{\text {fótons }} \mathrm{m}^{-2}\right) \mathrm{a}$

M. peruiferum: $\mathrm{y}=-\mathbf{3 8 , 0 0 8 x} 2+306,38 \mathrm{x}+703,87$;

$\mathrm{R} 2=0,9856 ; \int \Omega=17715,7\left(\mathrm{~mol}\right.$ fótons $\left.\mathrm{m}^{-2}\right) \mathrm{a}$

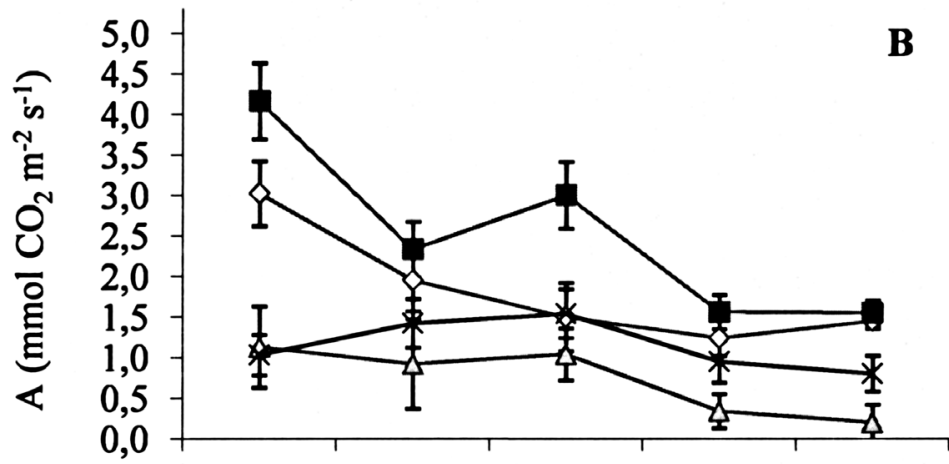

C. tomentosum: $\mathrm{y}=0,1959 \times 2-1,5597 \mathrm{x}+4,355$;

$\mathrm{R} 2=0,9955 ; \int \Omega=24,48\left(\mathrm{mmol} \mathrm{CO}_{2} \mathrm{~m}^{-2}\right) \mathrm{b}$

I. vera: $\mathrm{y}=0,1086 \mathrm{x} 2-1,2492 \mathrm{x}+5,0736$;

$\mathrm{R} 2=0,7775 ; \int \Omega=35,24\left(\mathrm{mmol} \mathrm{CO}_{2} \mathrm{~m}^{-2}\right) \mathrm{a}$

L. muehlbergianus: $\mathrm{y}=-0,0476 \mathrm{x} 2+0,0427 \mathrm{x}+1,1214 ; \mathrm{R} 2$

$=0,8676 ; \int \Omega=10,91\left(\mathrm{mmol} \mathrm{CO}_{2} \mathrm{~m}^{-2}\right) \mathrm{d}$

M. peruiferum: $\mathrm{y}=-0,128 \mathrm{x} 2+0,6743 \mathrm{x}+0,5315$;

$\mathrm{R} 2=0,788 ; \int \Omega=17,75\left(\mathrm{mmol} \mathrm{CO}_{2} \mathrm{~m}^{-2}\right) \mathrm{c}$

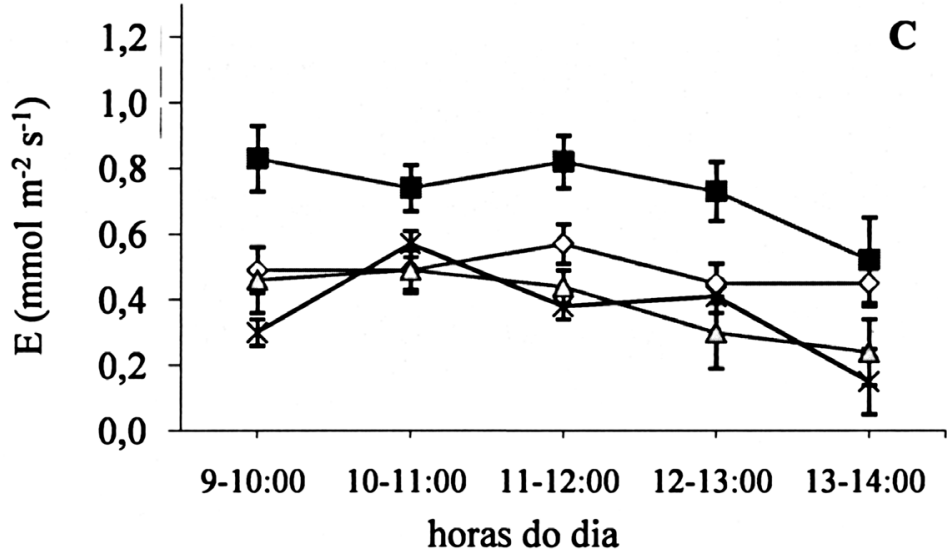

C. tomentosumy $=-0,0146 \times 2+0,0764 \mathrm{x}+0,4215$;

$\mathrm{R} 2=0,4243 ; \int \Omega=7,20\left(\mathrm{~mol} \mathrm{H}_{2} \mathrm{O} \mathrm{m}^{-2}\right) \mathrm{b}$

I. vera: $\mathrm{y}=-0,0301 \times 2+0,1191 \mathrm{x}+0,7052$;

$\mathrm{R} 2=0,8335 ; \int \Omega=10,82\left(\mathrm{~mol} \mathrm{H}_{2} \mathrm{O} \mathrm{m}^{-2}\right) \mathrm{a}$

L. muehlbergianus: $\mathrm{y}=-0,0206 \times 2+0,0623 \mathrm{x}+0,4272 ; \mathrm{R} 2$

$=0,9327 ; \int \Omega=5,78\left(\mathrm{~mol} \mathrm{H}_{2} \mathrm{O} \mathrm{m}^{-2}\right) \mathrm{c}$

M. peruiferum: $\mathrm{y}=-0,06162+0,3229 \mathrm{x}+0,0701$;

$\mathrm{R} 2=0,755 ; \int \Omega=5,79\left(\mathrm{~mol} \mathrm{H}_{2} \mathrm{O} \mathrm{m}^{-2}\right) \mathrm{c}$

horas do dia

Figura 3. Parâmetros fotossintéticos registrados no período de 9:00 à 14:00 h. Radiação Fostossintéticamante Ativa (PAR) (A), taxa fotossintética $(A)(B)$, taxa de transpiração $(E)(C)$ das espécies Centrolobium tomentosum $(\diamond)$, Inga vera $(\square)$, Lonchocarpus muehlbergianus $(\triangle)$ e Myroxylum peruiferum $(\times)$. Apresentam-se as equações polinomiais de $2^{\mathrm{a}}$ ordem e os correspondentes valores da integração das curvas de tendência. Cada ponto representa a média de 36 medidas (12/planta) realizadas no intervalo de $1 \mathrm{~h}$. 
consideram-se espécies tolerantes à sombra aquelas que não necessitam de clareiras para regenerar-se e que geralmente são espécies de estádios tardios de sucessão e espécies intolerantes à sombra, aquelas iniciais, que precisam de maior radiação solar para crescer (Hartshorn 1980). Embora M. peruiferum seja citada como tolerante à sombra (Lorenzi 1992), segundo os parâmetros de crescimento observados por Souza \& Válio (2001) e confirmados no presente trabalho, M.peruiferum seria uma espécie indiferente a condições de radiação plena ou sombreamento.

A espécie $M$. peruiferum foi a que apresentou a maior taxa de crescimento, a maior eficiência de uso de fotoassimilados e um bom aproveitamento do $\mathrm{N}$ orgânico, quando comparada com outras três espécies nativas da Mata Atlântica. Estas vantagens de significância ecológica lhes permitem rápida ocupação em espaços abertos e melhor aproveitamento dos recursos disponíveis no solo, o que torna esta espécie apta para serem utilizadas em projetos de revegetalização e recuperação de áreas degradadas.

\section{Agradecimentos}

À agencia CAPES pelo apoio financeiro.

\section{Literatura citada}

Antúnez, I., Retamosa, E.C. \& Villar, R. 2001. Relative growth rate in phylogenetically related deciduous and evergreen woody species. Oecologia 128: 172-180.

Arnon, D.J. 1949. Copper enzymes in isolated chloroplast polyphenoloxidases in Beta vulgaris. Plant Physiology 24: 1-15.

Ayres, M., Ayres Junior, M., Ayres, D.L. \& Santos, A.S. 2003. BioStat 3.0: Aplicações estatísticas nas áreas das ciências biológicas e médicas. Sociedade Civil Mamirauá, Belém.

Brown,S.M.\& Walsh, K.B. 1994. Anatomy of the legume nodule cortex with respect to nodule permeability. Australian Journal of Plant Physiology 21: 49-68.

Cancian, M.A.E. \& Cordeiro, L. 1998. Efeito do sombreamento no crescimento inicial de Lonchocarpus muehlbergianus Hassl. Acta Botanica Brasilica 12: 367-372.

Flemming, D.M. \& Gonçalves, M.B. 1992. Cálculo A: funções, limite, derivação, integração. 5 ed. Pearson Education do Brasil, São Paulo.

Garnier, E., Gobin, O. \& Poorter, H. 1995. Nitrogen productivity depends on photosynthetic nitrogen use efficiency and on nitrogen allocation within the plant. Annals of Botany 76: 667-672.
Gloser, V. \& Gloser, J. 1996. Acclimation capability of Calamagrostis epigejos and $C$. arundinaceae to changes in radiation environment. Photosynthetica 32: 202-212.

Grotkopp, E. \& Rejmánek, M. 2007. High seedling relative growth rate and specific leaf area are traits of invasive species: phylogenetically independent contrasts of woody angiosperms. American Journal of Botany 94: 526-532.

Hall, D.O., Scurlock, J.M., Bolhàr-Nordenkampf, H.R., Leegood, R.C. \& Long, S.P. 1993. Photosynthesis and production in a changing environment. A field and laboratory manual, Chapman \& Hall, London.

Hartshorn, G.S. 1980. Neotropical forest dynamics. Biotropica 12 (supplement 1): 23-30.

Hiscox, J.D. \& Israelstam, G.F. 1978. A method for the extraction of chlorophyll from leaf

tissue without maceration. Canadian Journal of Botany 57: 1332-1334.

Hoagland, D.R \& Arnon, D.I. 1950. The water culture method for growing plants without soils. California Agriculture Experimental Station Circular, Berkeley.

Hunt, R. 1982. Plant growth curves. The functional approach to plant growth analysis. Edward Arnold, London.

Ishida, A., Toma, T. \& Marjenah, A. 1999. Limitation of leaf carbon gain by stomatal and photochemical processes in the top canopy of Macaranga conifera, a tropical pioneer tree. Tree Physiology 19: 467-473.

James, J.J. \& Rebecca, E.D. 2007. A basis for relative growth rate differences between native and invasive forb seedlings. Rangeland Ecology \& Management 60: 395-400.

Kitajima, K. 1994. Relative importance of photosynthetic traits and allocation patterns as correlates of seedling shade tolerance of 13 tropical trees. Oecologia 98: 419-428.

Lambers, H. \& Poorter, H. 1992. Inherent variation in growth rate between higher plants: a search for physiological causes and ecological consequences. Advances Ecology Research 23: 187-261.

Lambers, H., Chapin, F.S. \& Pons, T.L. 1998. Plant physiological ecology. Springer-Verlag, New York.

Larcher, W. 2000. Ecofisiologia Vegetal. RiMa Editora, São Carlos.

Lichtenthaler, H.K. 1987. Chlorophylls and carotenoids: pigment of photosynthetic biomembranes. In: S.P. Colowick \& N.O. Kaplan (eds.). Methods in enzymology v. 148, Academic Press, New York, pp. 350-382.

Lorenzi, H. 1992. Árvores brasileiras: manual de identificação e cultivo de plantas arbóreas nativas do Brasil. Editora Plantarum, Nova Odessa.

Marañon, T. \& Grubb, J. 1993. Physiological basis and 
ecological significance of the seed size and relative growth rate relationship in Mediterranean annuals. Functional Ecology 7: 591-599.

Meziane, D. \& Shipley, B. 1999. Interacting components of interspecific relative growth rate: constancy and change under differing conditions of light and nutrient supply. Functional Ecology 13: 611-622.

Moreira, F.M., Silva, M.F. \& Faria, S.M. 1992. Ocurrence of nodulation in legume species in the Amazon region of Brazil. New Phytologist 121: 563-570.

Nandal, D.P., Rana, P. \& Kumar, A. 1999. Growth and yield of wheat (Triticum aestivum) under different tree spacings of Dalbergia sissoo based agriculture. Indian Journal of Agronomy 44: 256-260.

Poorter, H., Remkes, C. \& Lambers, H. 1990. Carbon and nitrogen economy of 24 wild species differing in relative growth rate. Plant Physiology 94: 621-627.

Ramakrishnan, P.S., Shukla, R.P. \& Boojh, R. 1982. Growth strategies of trees and their application to forest management. Current Science 51: 448-455.

Reich, P.B., Walters, M.B. \& Ellsworth, D.S. 1992. Leaf life-span in relation to leaf, plant, and stand characteristics among diverse ecosystems. Ecological Monographs 62: 365-392.

Schieving, F. \& Poorter, H. 1999. Carbon gain in a multispecies canopy: the role specific leaf area and photosynthetic nitrogen-use efficiency in the tragedy of the commons. New Phytologist 143: 201-211.

Schulze, E.D. \& Hall, A.E. 1982. Stomatal responses, water loss and $\mathrm{CO}_{2}$ assimilation rate in contrasting environments. In: O.L. Lange, P.S. Nober, C.B. Osmond \& H. Ziegler (eds.). Encyclopedia of plant physiology, v. 12B, Physiological plant ecology. II. Water relations and carbon assimilation. Springer Verlag, Berlin, pp.181-230.

Souza, R.P. \& Válio, I.F. 2001. Seed size, seed germination, and seedling survival of Brazilian Tropical tree species differing in sucessional status. Biotropica 33: 447457.

Wang, G.G., Qian, H. \& Klinka, K. 1994. Growth of Thuy a plicata seedlings along a light gradient. Canadian Journal of Botany 72: 1749-1747.

Witkowski, E.T. \& Lamont, B.B. 1991. Leaf specific mass confounds leaf density and thickness. Oecologia 88: 486-493.

Wright, I.J. \& Westoby, M. 2000. Cross-species relationships between seedling relative growth rate, nitrogen productivity and root $v s$ leaf function in 28 Australian woody species. Functional Ecology 14: 97-107.

Zelitch, I. 1982. The close relationship between net photosynthesis and crop yield. BioScience 32: 796802. 